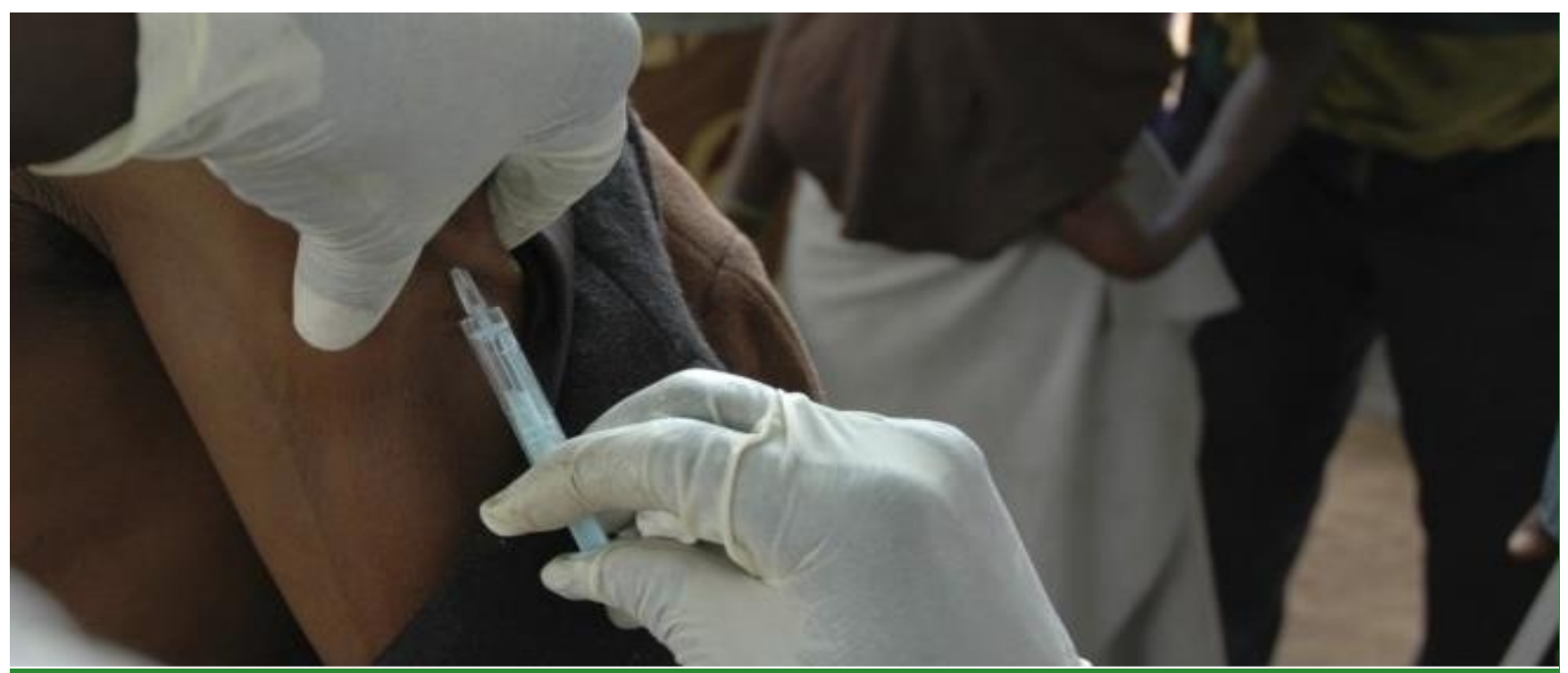

\title{
CABI's Global Health database - does it have a role to play in informing public health policy?
}

\section{Wendy Norris}

\section{Summary}

CABI's Global Health database has a significant role to play in informing public health policy helping tackle current and emerging health problems across the developing world. Systematic reviews, which are increasing in scope and number, are an excellent tool for evidence-based public health and have a growing influence on policy. However, for them to be meaningful they need to draw upon trustworthy data. Through interviews with researchers and through a literature review we found that CABI's Global Health database is able to offer significant support to systematic reviews, since it covers both print and electronic journals and offers unique content not available elsewhere. The Global Health database, through its use in specific systematic reviews, has contributed to IHME's (Institute for Health Metrics and Evaluation) Global Burden of Disease 2013 report and to a number of WHO clinical guidelines. Its use has informed the funding agenda of international donors, such as DFID and the Wellcome Trust, who are addressing research and practice in humanitarian crisis settings. The database has also been used in developing the research and planning agenda on trachoma for the charity Sightsavers. Moreover, we found that Global Health has a vital role to play in both disseminating locally conducted LMIC research within Africa and globally, by offering coverage of (local) papers in Africa and LMICs, and in nurturing collaboration between groups of researchers/projects.

\section{Introduction}

This case study assesses how the information captured in CABl's Global Health database is meeting CABl's mission of improving the lives of people worldwide. Global Health is the only specialist bibliographic abstracting and indexing database dedicated to public health. It completes the picture of international medical and health research by capturing key literature that is not covered by other databases, with $40 \%$ of the material contained in Global Health being unique to the database. 
Publications from over 100 countries in 50 languages are abstracted, and all relevant non-Englishlanguage papers are translated to give access to research not available elsewhere.

To find out how CABl's Global Health database influences public health policy we looked at its use by researchers drafting 'systematic reviews', which collate and analyse data relating to a particular area of medicine practice. Systematic reviews are popular with policymakers because they provide evidence on what drug, intervention or research gap to fund, and because they can also give insights into public knowledge and beliefs, which will affect whether a policy is likely to be effective. We spoke to a number of leading public health researchers around the world to see what use they made of Global Health when conducting systematic reviews. In particular we focused on the following topics:

1. the rise of chronic diseases in Africa

2. African research, and African and worldwide clinical practice

3. the influence of global burden of disease studies and WHO guidelines on policymakers and donors

4. the international research and funding agenda with respect to donors

\section{Rise of chronic diseases in Africa}

We met with a leading global health research group, Global Health Epidemiology Reference Group (GHERG), based at the University of Edinburgh, UK, which has recently undertaken a series of systematic reviews of chronic diseases in Africa using Global Health data. Dr Davies Adeloye, a senior lecturer in Demography, Health and Social Statistics at Covenant University, Ota, Nigeria, and a member of GHERG, explained that the Global Health database's coverage of papers on Africa and LMICs was vital in the preparation of GHERG's systematic reviews because it revealed unique sources of data, not available in other databases. These data sometimes provide a quite different picture to that which is normally presented.

For example, GHERG carried out a systematic review of hypertension in Africa ${ }^{1}$. They found that while a frequently quoted WHO African Region report states that 20 million people in Africa were living with hypertension in 2005, in fact the continent already had 92.3 million cases in 2000. GHERG concluded, based in part on data from Global Health, that hypertension prevalence is underestimated and is increasing. According to Dr Adeloye unique data from Global Health helped to provide him and his fellow researchers with this more accurate picture of hypertension in Africa. As he explained, GHERG has a "research policy to always check the Global Health database to provide wider capture of relevant articles on global health, which may not be indexed by other databases."

\section{African research and clinical practice}

\section{African research}

Dr Adeloye told us that he recommends the Global Health database to colleagues and students engaged in research in, and about, Africa because its coverage is wider than other databases/publishing platforms. According to Adeloye: "most journal websites in LMIC settings [including in Africa] are not fully operational, with articles mainly available only in print form". As a result, many journal publishing platforms fail to cover research from these sources. By contrast, Global Health covers print journals, thus providing a much fuller picture of African research. In a context in which funders are now moving to invest in the dissemination of locally conducted research within Africa (and globally), Global Health, with its better coverage of African print journals, has a significant role to play in the dissemination of African research.

\section{African clinical practice}

A researcher who has used the Global Health database to conduct systematic reviews of clinical and public health practice in the African context is Dr Peter MacPherson, a public health lecturer at the University of Liverpool, UK, who explained to us that he used data from the database in a series of reviews of TB and HIV treatment in sub-Saharan Africa. These reviews contained significant findings 
with clear policy implications. On TB, MacPherson showed that in Africa $18 \%$ of adults diagnosed with infectious TB are lost to follow-up before they start treatment ${ }^{2}$, and with HIV infections he showed that a high proportion of people who should receive ART based on CD4 counts are not doing so because of test inaccuracy or unavailability ${ }^{3}$; and also that there is a paucity of data on HIV virological outcomes for adolescents receiving antiretroviral therapy ${ }^{4}$.

\section{Worldwide clinical practice}

Impact of the Global Health database is not restricted solely to the African context; the WHO's guidelines, which are applicable worldwide, are often based on research covered by and accessed through this database. For example:

- the WHO's 2016 updated guidelines for ART will include recommendations based on MacPherson's systematic reviews of HIV treatment (see above)

- the WHO's guidelines on task-shifting to health workers in the area of safe abortion and contraception ${ }^{5}$ reviewed studies found in Global Health

- Global Health was a key source of data for the WHO's 2011 guidelines for the programmatic management of drug-resistant TB data ${ }^{6}$

\section{Influence of global burden of disease studies and WHO guidelines on policymakers and donors}

The impact of Global Health is also evident through its ability to provide more accurate data on the global burden of disease. Policymakers currently rely on burden of disease studies, such as the WHO guidelines or the IHME's regular Global Burden of Disease reports, to make country policy and to decide on allocation of development funds and emergency aid. However, these studies are not always accurate (relying on estimates based on advanced statistical models) or fully up to date and sources of data may not be transparent. For example, according to Dr. Adeloye, burden of disease estimates for Africa provided by the IHME may not truly reflect the burden in this setting as "there is a major lack of data at regional and national levels". More current and complete data can be provided by drawing upon the Global Health database, leading to more accurate disease studies, and thus improved policy.

An example of the role of Global Health in this regard is seen in the recent systematic reviews of the impact of water, sanitation and hygiene (WASH) on preventing water-borne diseases, published by an expert group of scientists from 14 collaborating institutions brought together by the WHO.

Global Health played a key role in conducting these systematic reviews of WASH. Referring to her review on drinking water and sanitation interventions ${ }^{7}$, Dr Jennyfer Wolf (lead author) said that "as the evidence is limited (especially for sanitation) all evidence that met our inclusion criteria was very important for us". All of the studies analysed are in Global Health, including four (Fan and Ajay ${ }^{8}$; Pradhan and Rawlings ${ }^{9}$; Begum S. et $\mathrm{al}^{10}$; Galiani S, et $\mathrm{al}^{11}$ ) not recorded elsewhere (in Medline or Embase).

The impact of the reviews authored by Dr Wolf (drinking water and sanitation) and Dr. Freeman (hygiene and handwashing ${ }^{12}$ ) and their colleagues, drawing on data sourced from Global Health, has been substantial: they formed part of the 2014 WHO report Preventing diarrhea through better water, sanitation and hygiene (exposures \& impacts in low- and middle-income countries ${ }^{13}$ ) and were also acknowledged by IHME in their 2013 Global Burden of Disease report ${ }^{14}$. Dr Val Curtis, a co-author on both WASH reviews and WHO report, described how the reviews were part of a larger body of work which had led to, for the first time, handwashing practices being accepted by IHME as a separate risk factor for disease. From 2013 onwards, all IHME reports will feature this.

In addition, Dr Curtis stated that the reviews (and WHO report) now form part of the evidence base for WASH. In this context, they had an impact on Sustainable Development Goal 6 on universal access to water and sanitation, which was amended to include a handwashing indicator i.e. to count as an adequate sanitation unit, it must now have a handwashing facility attached. Through her advocacy roles, data from the reviews will support the Indian government's 'Clean India' 
campaign, which seeks to eliminate open defecation by 2019, and Unilever Lifebuoy soap's global handwashing campaign ("helping 1 billion people around the world improve their handwashing habits by 2020 ").

\section{International research and funding agenda of donors}

A good example of the database's impact in this area is through the Enhancing Learning and Research for Humanitarian Assistance's 'Research for Health in Humanitarian Crises' (R2HC) programme, funded by the Wellcome Trust and DFID, which aims to increase the level and quality of research on public health and emergency responses to humanitarian crises, and to create an evidence base regarding what works best in such situations

Dr Bayard Roberts and Dr Anita Ramesh of London School of Hygiene and Tropical Medicine (LSHTM) and researchers on R2HC gave us insight into the use of Global Health in the first stage of the R2HC process: a system- wide Humanitarian Health Evidence Review (HHER ${ }^{15}$, completed 2015), which investigated the research landscape across seven different streams over the past 30 years to identify the key challenges and needs faced by practitioners in crisis situations. Papers for individual health streams were published throughout 2015, culminating in the full report.

"The HHER was the first comprehensive review of its kind that looked at gaps in the evidence base on effectiveness of health responses in humanitarian crises", said Dr Bayard Roberts, a senior lecturer in health systems and policy at the School, and who as co-principal investigator with $\mathrm{Dr}$ Karl Blanchet, led the entire evidence review. "HHER was the first part, and an important part, of the R2HC programme, setting the research and funding agenda for DFID \& Welcome Trust in humanitarian health settings. The 2 nd part, which was always the programme's end point, is to facilitate \& fund collaborations between academics and operational agencies, to conduct research into the research gaps and needs we identified."

Global Health was used for all 9 systematic reviews conducted by the LSHTM team in the evidence review. Dr Roberts commented that this was because "it is the norm for systematic reviewers at the LSHTM to include the Global Health database in order to be comprehensive." As one of the main bibliographic databases for gathering information, he recommends it to students for summer projects and systematic reviews.

Dr Ramesh, an infectious disease epidemiologist spoke to us about one of these systematic reviews. Published in the journal PLOS ONE, 2015 ${ }^{16}$, it focused on health outcomes associated directly to WASH interventions but found that of 3963 studies retrieved, only 6 had measured a health outcome and that indirectly through a measure of water quality. Global Health is more relevant to WASH research than Medline and Embase because WASH is not a medicalized area of research for, as her paper points out, "typically the WASH sector has largely been led by water and sanitation engineers rather than medical professionals". Separately from the R2HC review, Dr Ramesh with other colleagues at LSHTM were commissioned by Sightsavers (the largest eye charity in the world) to carry out two systematic reviews on the effect of climate change on trachoma ${ }^{17}$, a neglected tropical disease that causes blindness, and on the ecology of the trachoma vector, Musca sorbens ${ }^{18}$. Sightsavers needed this information in order to plan its long term strategy "to step-up global efforts to eliminate trachoma, before regional climate shifts makes the current situation worse."[to quote Dominic Haslam, Director of Policy at Sightsavers and coauthor of both studies, commenting on the publication of the vector paper for LSHTM news ]. Global Health was particularly important for the vector ecology study, linking environmental and entomological information in order to predict changes in future distribution.

\section{Key points}

Global Health database is seen by major global health research groups as a key resource for systematic reviews on low- and middle-income countries (LMICs), particularly in Africa. It:

- provides unique content for systematic reviews, thus informing public health policy 
- contributed to the Institute for Health Metrics and Evaluation's (IHME) Global Burden of Diseases, Injuries and Risk Factors Study 2013, and to a number of World Health Organization (WHO) clinical guidelines

- informed the funding agenda of international donors addressing research and practice for humanitarian crisis settings

- was used in developing the research agenda of a charity

\section{References}

1 Estimating the prevalence and awareness rates of hypertension in Africa: a systematic analysis. Adeloye, D.; Basquill, C.; PLoS ONE, 2014.

2 Pre-treatment loss to follow-up in tuberculosis patients in low- and lower-middle-income countries and high-burden countries: a systematic review and meta-analysis. MacPherson et al. Bulletin of the World Health Organization, 2014

3 Diagnostic accuracy of the WHO clinical staging system for defining eligibility for ART in subSaharan Africa: a systematic review and meta-analysis. Munthali C. et al. Journal of the International AIDS Society, 2014

4 Viral suppression in adolescents on antiretroviral; treatment: review of the literature and critical appraisal of methodological challenges. Ferrand et al. Tropical Medicine \& International Health, 2016

5 WHO's guidelines on task-shifting to health workers in the area of safe abortion and contraception

6 WHO's 2011 guidelines for the programmatic management of drug-resistant TB data

7 Systematic review: assessing the impact of drinking water and sanitation on diarrhoeal disease in low- and middle-income settings: systematic review and meta-regression. Wolf et al. Tropical Medicine \& International Health, 2014.

8 What prevents child diarrhoea? The impacts of water supply, toilets, and hand-washing in rural India Fan, Y. M. V and Ajay, M. Journal of Development Effectiveness, 2011

9 The Impact and Targeting of Social Infrastructure Investments: Lessons from the Nicaraguan Social Fund. Pradhan, M. and Rawlings, L. World Bank Economic Review, 2002

10 Do Water and Sanitation Interventions Reduce Childhood Diarrhoea? New Evidence from Bangladesh. Begum S. et al. Bangladesh Development Studies, 2012

11 Water Expansions in Shantytowns: Health and Savings. Galiani, S. et al. Economica 2009

12 Systematic review: hygiene and health: systematic review of handwashing practices worldwide and update of health effects. Freeman et al. Tropical Medicine \& International Health 2014.

13 Preventing diarrhoea through better water, sanitation and hygiene: exposures and impacts in low- and middle-income countries. WHO report 2014

14 Global, regional, and national comparative risk assessment of 79 behavioural, environmental and occupational, and metabolic risks or clusters of risks in 188 countries, 1990-2013: a systematic analysis for the Global Burden of Disease Study 2013. [GBD risk factors collaborators] Forouzanfar, M. H et al. , Lancet (British edition), 2015

15 An evidence review of research on health interventions in humanitarian crises Blanchet $\mathrm{K}$. et al. 2015

16 Evidence on the effectiveness of water, sanitation, and hygiene (WASH) interventions on health outcomes in humanitarian crises: a systematic review. Ramesh et al. PLOS ONE 2015

17 The impact of climatic risk factors on the prevalence, distribution, and severity of acute and chronic trachoma. Ramesh et al. PLOS Neglected Tropical Diseases 2013 
18 The impact of climate on the abundance of Musca sorbens, the vector of trachoma. Ramesh et al. Parasites \& Vectors 2016

\section{Acknowledgements}

$\mathrm{CABI}$ is an international intergovernmental organisation, and we gratefully acknowledge the core financial support from our member countries (and lead agencies) including the United Kingdom (Department for International Development), China (Chinese Ministry of Agriculture), Australia (Australian Centre for International Agricultural Research), Canada (Agriculture and Agri-Food Canada), Netherlands (Directorate-General for International Cooperation), and Switzerland (Swiss Agency for Development and Cooperation).

\section{Author}

Wendy Norris, CABI

\section{How to cite this paper}

Norris, W.E. 2016. CABl's Global Health database - does it have a role to play in informing public health policy? CABI Study Brief 19. DOI: http://dx.doi.org/10.1079/CABICOMM-64-1702 